\title{
Antiplatelet therapy for prevention of hemodialysis vascular access thrombosis and improving survival
}

\author{
Matteo Tozzi ${ }^{1}$ (D) Maurizio Gallieni ${ }^{2,3}$
}

Received: 30 December 2018 / Accepted: 2 March 2019 / Published online: 19 March 2019

(c) Italian Society of Nephrology 2019

Stenosis and occlusion are common and critical complications of arterio-venous dialysis access and the goal of improving access patency often remains elusive for nephrologist and surgeons. Among the drugs used for reducing the rate of access thrombosis in hemodialysis patients, surely the most debated are the antiplatelet drugs, whose data in the literature are limited and provide conflicting results. In 1994, the Antiplatelet Trialists Collaboration [1] analyzed the results of 60 randomized controlled trials (RCT) studying about 8000 patients at varying degrees of risk of coronary or peripheral artery occlusion, showing that antiplatelet therapy (APT), mainly aspirin alone or aspirin plus dipyridamole, greatly reduced the risk of vascular occlusion. The study also included 400 dialysis patients with a shunt or fistula placed for hemodialysis access and occlusion was $17 \%$ with antiplatelet therapy versus $39 \%$ in controls. This resulted in an increased use of APT, but subsequent studies did not give consistent results.

The paper by Locham et al. published in this issue of the Journal of Nephrology [2] highlights with a solid sample size the role of antiplatelet therapy on patency of dialysis vascular access (78\% AV fistulae). About 25,000 hemodialysis patients from a large dialysis vascular qualitative initiative database were included and divided into no-APT versus any APT (aspirin or P2Y12 inhibitors). The primary endpoint of the study was loss of primary patency at 12 months, a good time-frame to assess thrombotic complications. In the AV grafts arm, patency was statistically higher in patients receiving APT. In the analysis of treatment

Matteo Tozzi

matteo.tozzi@uninsubria.it

1 Vascular Surgery Unit, Department of Medicine and Surgery, University of Insubria, Varese, Italy

2 Nephrology and Dialysis Unit, ASST Fatebenefratelli Sacco, Milano, Italy

3 Department of Clinical and Biomedical Sciences 'Luigi Sacco', University of Milano, Milan, Italy subgroups, P2Y12 receptor blockers (such as clopidogrel and ticlopidine) were more effective in reducing patency loss than ASA alone. Compared to no-APT, multivariate analysis showed that the loss of primary patency at 12 months was $13 \%$ lower in ASA users and 24\% lower in P2Y12 receptor blockers users. On the other hand, in patients with AV fistulae primary patency was not modified by APT compared to controls. Therefore, this study suggests that patients who receive prosthetic vascular access should be treated with P2Y12 receptor blockers. Importantly, in this observational study the issue of patients at risk of bleeding was not considered and this category of patients should be excluded from this therapy until more robust safety data are available.

How do these finding compare with previous evidence? We should consider different situations, such as the effects of APT on AV fistulae or grafts and short term versus medium to long-term outcomes.

In 2008, a RCT failed to demonstrate a protective effect of clopidogrel on early failure of AV fistulae [3]. Although fistula thrombosis occurred in $12.2 \%$ of participants assigned to clopidogrel compared with $19.5 \%$ assigned to placebo (relative risk, 0.63 ), suitability for dialysis at 3 months did not differ between the clopidogrel and placebo groups (relative risk, 1.05). However, lack of maturation was remarkably and unexpectedly high in both groups, $38 \%$ vs $40 \%$, respectively [3]. Thus, the results of this study discouraged the use of APT, because clopidogrel reduced the frequency of early thrombosis of new arteriovenous fistulas but did not increase the proportion of fistulas that became suitable for dialysis. Similar conclusions were reached by the Cochrane review published in 2015 [4] including RCTs of active drugs versus placebo in dialysis patients with an AV access. The meta-analyses of three studies for ticlopidine, with a short follow-up of only 1 month, suggested that ticlopidine may be beneficial as an adjuvant treatment to increase the patency of $\mathrm{AV}$ fistulae and grafts in the short term, while there was insufficient evidence to determine differences in graft patency 
between placebo and other treatments such as aspirin, fish oil, clopidogrel, PRT-201, dipyridamole, dipyridamole plus aspirin, warfarin, and sulphinpyrazone. The presence of methodological issues and small study sizes indicated that further studies be undertaken to assess the use of these anti-platelet drugs in renal patients with an arteriovenous fistula or graft [4].

Murley et al. [5] studied the effect of antithrombotic agents on early and late fistula failure through a retrospective analysis of two prospectively maintained databases of access operations and dialysis sessions of 671 patients. Antithrombotic medication did not have an effect on AVF survival and primary failure was similar (18-19\%) between patients on antiplatelet, anticoagulants or no antithrombotic medication. On the other hand, patients already on antithrombotic medication had no increase in bleeding-related cannulation adverse events, suggesting that patients can continue taking them without increasing the risk of interrupted dialysis [5].

In contrast, oral anticoagulation does not appear to confer protection from thrombosis and it increases the risk of bleeding. A RCT assessed low dose warfarin in AV grafts, targeting to an international normalized ratio of 1.4-1.9. This therapy did not decrease graft thrombosis, and the study was terminated early for major bleeding complications in the treatment group [6]. This finding is supported by results of studies on the treatment of atrial fibrillation with warfarin in older adults with end stage renal disease, who present a major risk of bleeding complications with early discontinuation of therapy [7].

An RCT assessed the combination of long-term daily clopidogrel $(75 \mathrm{mg})$ and aspirin $(325 \mathrm{mg}$ ) versus placebo for prevention of $\mathrm{AV}$ access graft thrombosis was prematurely stopped after 12 months because of a significantly increased risk of bleeding among the intervention group [8]. Moreover, there was no significant benefit of active treatment in the prevention of thrombosis. Based on this study, double APT is considered at high risk of bleeding in dialysis patients and it should not be considered for prevention of access thrombosis.

Dixon et al. [9] enrolled and randomized 1056 patients to ASA plus dipyridamole versus placebo, with a significant but modest effect in reducing the risk of stenosis and improving the duration of primary unassisted patency of newly created grafts. The incidence of primary unassisted graft patency at 1 year indicated an absolute reduction of $5 \%$ (patency $28 \%$ active treatment versus $23 \%$ placebo) and a relative reduction of $18 \%$. This was associated with a significantly prolonged duration of primary unassisted patency and graft stenosis inhibition. However, the DAC graft studies were characterized by a marked overall burden of graft failure, with over $75 \%$ of patients requiring an intervention to maintain patency within the first year after graft placement. In addition, the secondary outcomes (incidence of cumulative graft failure and death) did not differ significantly between study groups.

Compared with the general population, dialysis patients show an increased atherosclerotic events and bleeding risk. Therefore, when considering APT for reduction of cardiovascular mortality or for improving vascular access patency, in CKD patients, it is of the utmost importance keeping in mind that the individual patient might develop severe bleeding complications. Such concept is supported by a systematic review indicating that APT increases the risk of major bleeding by $33 \%$ and minor bleeding by $48 \%$ in CKD patients [10].

The study of Locham et al. [2] also found an increased risk of death for no-APT patients, indicating either a selection bias of patients or a protective effect of APT on mortality. After adjusting for patients' characteristics, patients on no-APT had a 12-fold increased risk of in-hospital mortality compared to patients on any APT. The mortality rate was significantly lower in the APT arm in both AVG $(0.15$ versus $0.7 \%)$ and $\operatorname{AVF}(0.04 \%$ vs $0.4 \%)$ patients. A selection bias could be hypothesized, but patients discharged with APT (49\% of AVG and 46\% AVF) had more comorbidities compared to No-APT patients. Patients on APT were also older in both AVG (65 versus 61 years) and AVF (64 versus 57 years) groups. These differences could reflect a more aggressive approach to patients at higher risk of cardiovascular disease, which is a common indication for the administration of APT. Thus, it appears even more convincingly that APT might reduce the mortality of patients undergoing the creation of hemodialysis vascular access, because APT patients were more at risk.

Systematic reviews and clinical practice guidelines, which are mainly based on randomized clinical trials results, offer contrasting advices on the use of ATP for prevention of hemodialysis access thrombosis. In most cases, caution is advised, because even when a reduction in the risk of thrombosis is observed, the adverse effects burden is uncertain, especially regarding bleeding and overall mortality. Different suggestions can also be found in guidelines regarding AVF and AVG and there is clearly a need for more studies to clearly indicate how to translate study results into clinical practice. For example, the recent guidelines of the European Society of Vascular Surgery advise against the use of APT, stating that "long-term anti-thrombotic therapy should not be used to prolong vascular access patency in hemodialysis patients". With the current methodology of practice guidelines production, the large observational study by Locham et al. [2] will not affect recommendations, but it give us the possibility of designing new RCT which hopefully will determine the real value of APT in vascular access. Considering the higher thrombosis rate of AVG compared to AVF and the findings of Locham et al., P2Y12 inhibitors could reduce the thrombosis gap in patients with a prosthetic 
vascular access. The study by Locham et al. is unique in reporting on a medical intervention that provides not only an improvement in vascular access graft outcomes but also on an improved patient survival, which implies a low risk of adverse events. This study points to the adoption of P2Y12 inhibitors in patients subjected to the creation of a prosthetic vascular access who are not at high bleeding risk, while no long-term advantages are expected for patients with AVF. However, the overall effect on improved patient survival suggests that long term APT should be further studied in all hemodialysis patients.

\section{Compliance with ethical standards}

Conflict of interest On behalf of all authors, the corresponding author states that there is no conflict of interest.

Ethical approval This article does not contain any studies with human participants performed by any of the authors.

\section{References}

1. Antiplatelet Trialists' Collaboration (1994) Collaborative overview of randomised trials of antiplatelet therapy-II: maintenance of vascular graft or arterial patency by antiplatelet therapy. BMJ 308:159-168

2. Locham S, Beaulieu RJ, Dakour-Aridi H, Nejim B, Malas MB (2018) Role of antiplatelet therapy in the durability of hemodialysis access. J Nephrol 31(4):603-611

3. Dember LM, Beck GJ, Allon M, Delmez JA, Dixon BS, Greenberg A, Himmelfarb J, Vazquez MA, Gassman JJ, Greene T, Radeva MK, Braden GL, Ikizler TA, Rocco MV, Davidson IJ, Kaufman JS, Meyers CM, Kusek JW, Feldman HI, Dialysis Access Consortium Study Group (2008) Effect of clopidogrel on early failure of arteriovenous fistulas for hemodialysis: a randomized controlled trial. JAMA 299:2164-2171. https://doi.org/10.1001/ jama.299.18.2164

4. Tanner NC, Da Silva A (2015) Medical adjuvant treatment to increase patency of arteriovenous fistulae and grafts. Cochrane Database Syst Rev 7:CD002786. https://doi.org/10.1002/14651 858.CD002786.pub3

5. Murley A, Wijewardane A, Wilmink T, Baharani J (2016) Should patients be on antithrombotic medication for their first arteriovenous fistulae? J Vasc Access 17:118-123. https://doi. org/10.5301/jva.5000491

6. Crowther MA, Clase CM, Margetts PJ, Julian J, Lambert K, Sneath D, Nagai R, Wilson S, Ingram AJ (2002) Low-intensity warfarin is ineffective for the prevention of PTFE graft failure in patients on hemodialysis: a randomized controlled trial. J Am Soc Nephrol 13:2331-2337

7. Tan J, Bae S, Segal JB, Zhu J, Segev DL, Alexander GC, McAdams-DeMarco M (2017) Treatment of atrial fibrillation with warfarin among older adults with end stage renal disease. J Nephrol 30:831-839

8. Kaufman JS, Connor TZ, Zhang JH, Cronin RE, Fiore LD, Ganz MB, Goldfarb DS, Peduzzi PN, Veterans Affairs Cooperative Study Group on Hemodialysis Access Graft Thrombosis (2003) Randomized controlled trial of clopidogrel plus aspirin to prevent hemodialysis access graft thrombosis. J Am Soc Nephrol 14:2313-2321

9. Dixon BS, Beck GJ, Vazquez MA, Greenberg A, Delmez JA, Allon M, Dember LM, Himmelfarb J, Gassman JJ, Greene T, Radeva MK, Davidson IJ, Ikizler TA, Braden GL, Fenves AZ, Kaufman JS, Cotton JR Jr, Martin KJ, McNeil JW, Rahman A, Lawson JH, Whiting JF, Hu B, Meyers CM, Kusek JW, Feldman HI, DAC Study Group (2009) Effect of dipyridamole plus aspirin on hemodialysis-graft patency. N Engl J Med 360:2191-2201. https://doi.org/10.1056/NEJMoa0805840

10. Migliori M, Cantaluppi V, Scatena A, Panichi V (2017) Antiplatelet agents in hemodialysis. J Nephrol 30:373-383

Publisher's Note Springer Nature remains neutral with regard to jurisdictional claims in published maps and institutional affiliations. 